\title{
The Hartle-Hawking State for the Bianchi IX Model in Supergravity
}

\author{
R. GRAHAM \\ Fachbereich Physik \\ Universität Gesamthochschule Essen \\ D-45117 Essen \\ Germany \\ H. LUCKOCK \\ School of Mathematics and Statistics \\ University of Sydney \\ NSW 2006 \\ Australia
}

\begin{abstract}
We solve the quantum constraints for homogeneous $\mathrm{N}=1$ supergravity on 3geometries with a Bianchi IX metric. Because these geometries admit Killing vectors with the same commutation relations as the angular momentum generators, there are two distinct definitions of homogeneity. The first of these is well-known and has been shown by D'Eath to give the wormhole state. We show that the alternative definition of homogeneity leads to the Hartle-Hawking "no boundary" state.

PACS numbers: $98.80 . \mathrm{H}, 04.65$
\end{abstract}


Minisuperspace models have attracted interest for a long time as a useful testing ground for new ideas on the many unresolved questions in quantum gravity [1, 2, 31. Recently a variety of minisuperspace models have been shown to admit simple supersymmetric extensions [4, 5, 6, 7, 8, 9 and it appears that many of these can be derived from a full supergravity theory by dimensional reduction of the classical theory 四, 5].

A slightly different approach is to start with the supersymmetry constraints for full supergravity and then to consider only homogeneous configurations of the metric and the fields. This approach has been used recently by D'Eath to quantise Bianchi IX cosmologies [10] (see also [11]). D'Eath showed that if the components of the Rarita-Schwinger field in an invariant basis are required to be spatially constant, then the supersymmetry constraints can be solved by a wormhole state but, surprisingly, not by a Hartle-Hawking state.

This result is puzzling, as it calls into question the existence of a Hartle-Hawking state for homogeneous $N=1$ supergravity. The aim of this letter is to solve this puzzle. We will show that the existence of a Hartle-Hawking state depends on the ansatz used for the fermion fields; specifically, on whether the spinor components have the same or opposite sign at antipodal points of the spatial 3-manifold.

The spatial 3-geometry in Bianchi IX models is homeomorphic either to $S O(3)$ or to its double covering $S U(2) \sim \mathcal{S}^{3}$. The second choice is necessary if we seek a homogeneous Hartle-Hawking state since $S U(2)$ (unlike $S O(3)$ ) can be continuously shrunk to a point within some smooth 4-manifold.

Identifying points in $\mathcal{S}^{3}$ with matrices $U \in S U(2)$, the symmetry transformations for homogeneous geometries can be represented by mappings $U \mapsto U B^{\dagger}$ where $B \in$ $S U(2)$. A field $\phi$ can be said to be homogeneous if it is invariant, up to the action of some locally-acting group, under these transformations; in other words, if it satisfies

$$
\phi\left(U B^{\dagger}\right)=R_{B} \phi(U)
$$


where $R_{B}$ is some representation of the matrix $B \in S U(2)$.

The most obvious definition of homogeneity arises from the trivial representation, in which $R_{B}=1$ for all $B \in S U(2)$; this choice leads to D'Eath's ansatz, in which all fields have constant components. However another choice is possible, since physical fields carry a representation of the group of spatial rotations which are themselves represented by elements of $S U(2)$. An alternative definition of homogeneity can thus be obtained by choosing $R_{B}$ to be the rotation represented by the matrix $B$ (or by matrices obtained from $B$ by unitary transformations).

The distinction between the two kinds of homogeneity is clearest in the case of a spin- $\frac{1}{2}$ field $\chi$ on $\mathcal{S}^{3}$. Instead of requiring $\chi$ to have constant components when referred to an invariant dreibein (i.e. a dreibein invariant under the diffeomorphisms $U \mapsto U B^{\dagger}$, we can demand that $U \chi$ should be constant on $\mathcal{S}^{3}$.

For our purposes, it will be useful to obtain a differential version of the new homogeneity condition. The spatial 3-geometry of the Bianchi IX model admits three Killing vector fields $\mathbf{K}_{p}$ and three invariant 1-forms $\tilde{\boldsymbol{\omega}}^{p}$ satisfying

$$
\left[\mathbf{K}_{p}, \mathbf{K}_{q}\right]=C_{p q}^{r} \mathbf{K}_{r}, \quad \tilde{\boldsymbol{d}} \tilde{\boldsymbol{\omega}}^{p}=\frac{1}{2} C^{p}{ }_{q r} \tilde{\boldsymbol{\omega}}^{q} \wedge \tilde{\boldsymbol{\omega}}^{r} \quad C^{r}{ }_{p q}=\epsilon_{p q s} \delta^{s r}
$$

while the 3 -metric has the form $h=h_{p q} \tilde{\boldsymbol{\omega}}^{p} \otimes \tilde{\boldsymbol{\omega}}^{q}$. The components of $h$ in the invariant basis can be written as

$$
h_{p q}=e^{2 \beta_{a}} \mathcal{O}_{p}{ }^{a} \mathcal{O}_{q}{ }^{a}
$$

where the diagonalising matrix $\mathcal{O}_{q}{ }^{a}$ satisfies the orthogonality condition

$$
\delta^{p q} \mathcal{O}_{p}^{a} \mathcal{O}_{q}^{b}=\delta^{a b}, \quad \operatorname{det} \mathcal{O}=1
$$

Apart from inversions and relabellings of the axes, there is a unique matrix $\mathcal{O}$ and a unique set of scale parameters $\beta_{a}$ corresponding to a given 3-metric $h$.

The three scale parameters $\beta_{a}$ represent the physical degrees of freedom of the 3-metric. On the other hand, the three degrees of freedom contained in the matrix 
$\mathcal{O}$ are pure gauge, and are associated with the group of diffeomorphisms generated by the three Killing vector fields dual to the 1 -forms $\tilde{\boldsymbol{\omega}}^{p}$. By taking the matrix $\mathcal{O}$ to be fixed, we eliminate these unphysical degrees of freedom.

We can now identify a preferred Lorentz frame in which the axes of the dreibein coincide with the main axes of the metric tensor; in this special gauge the dreibein consists of the three 1-forms

$$
\tilde{\boldsymbol{e}}^{a}=e^{a}{ }_{p} \tilde{\boldsymbol{\omega}}^{p}, \quad e^{a}{ }_{p}=e^{\beta_{a}} \mathcal{O}_{p}{ }^{a} .
$$

We will work exclusively in this preferred frame, thereby eliminating the gauge degrees of freedom associated with Lorentz transformations.

In the classical theory, the 3-geometry is embedded in a 4-dimensional spacetime equipped with a vielbein consisting of four 1-forms which we take as

$$
{ }^{(4)} \tilde{\boldsymbol{e}}^{0}=N \tilde{\boldsymbol{d}} t, \quad{ }^{(4)} \tilde{\boldsymbol{e}}^{a}=e^{a}{ }_{p}\left(\tilde{\boldsymbol{\omega}}^{p}+N^{p} \tilde{\boldsymbol{d}} t\right) \quad(a=1,2,3) .
$$

We are entitled to impose a coordinate condition on the lapse function $N$, in addition to the three we have already imposed by fixing the matrix $\mathcal{O}$. Together with the three scale parameters $\beta_{a}$, the components $N^{p}$ of the shift vector then account for the six physical degrees of freedom of the 4-metric. Note however that the wave function is independent of $N$ and the variables $N^{p}$, and so these do not appear in the quantum theory which we consider here. We are therefore interested only in the intrinsic geometry of the spatial 3-manifold, which is fully described by the dreibein $\tilde{\boldsymbol{e}}^{a}$.

In the preferred Lorentz frame, the new homogeneity condition for an arbitrary field $\phi$ defined on the spatial $\mathcal{S}^{3}$ manifold has the form

$$
£_{\mathbf{K}_{p}} \phi=-i\left(i_{\mathbf{K}_{p}} \tilde{\boldsymbol{\omega}}^{q}\right) \mathcal{O}_{q}{ }^{a} \mathbf{J}^{a} \phi \quad p=1,2,3
$$

where $i_{\mathbf{K}_{p}} \tilde{\boldsymbol{\omega}}^{q}$ denotes the interior product of $\mathbf{K}_{p}$ with $\tilde{\boldsymbol{\omega}}^{q}$, and $£_{\mathbf{K}_{p}}$ is the Lie derivative along $\mathbf{K}_{p}$. (The dreibein is invariant under the action of the $\mathbf{K}_{p}$ and so $£_{\mathbf{K}_{p}}$ does 
not contain a spin connection[12].) The $\mathbf{J}^{a}$ generate physical rotations of $\phi$ about the axes defined by the 1 -forms $\tilde{\boldsymbol{e}}^{a}$ and obey the standard commutation relations

$$
\left[\mathbf{J}^{a}, \mathbf{J}^{b}\right]=i \epsilon^{a b c} \mathbf{J}^{c}
$$

These relations and the orthogonality of the matrix $\mathcal{O}$ ensure that the new homogeneity condition is integrable and admits non-trivial solutions.

Condition (2) implies that $\phi$ rotates through an angle $2 \pi$ relative to an invariant frame as one follows a path between antipodal points on $\mathcal{S}^{3}$. In particular, this means that the components of any homogeneous spinor field will have opposite signs at antipodal points.

Since the operators appearing on both sides of (2) obey the Leibnitz rule, it is clear that products of homogeneous fields will themselves be homogeneous. The 3 -metric $h$ is also homogeneous since $£_{\mathbf{K}_{p}} h=0=\mathbf{J}_{q} h$.

Of particular interest in supergravity is the spatial part $\psi^{A}=\psi^{A a} \tilde{\boldsymbol{e}}^{a}$ of the spin- $\frac{3}{2}$ Rarita-Schwinger field which appears in the quantum supersymmetry constraints. Introducing local coordinates $x^{i}$ on the 3 -manifold, with $\tilde{\boldsymbol{\omega}}^{p}=\omega^{p}{ }_{i} \tilde{\boldsymbol{d}} x^{i}$, the homogeneity conditions for the components $\psi^{A a}$ can be written as

$$
\frac{\partial \psi^{A a}}{\partial x^{i}}=-i \omega^{p}{ }_{i} \mathcal{O}_{p}{ }^{b}\left(\frac{1}{\sqrt{2}} \sigma_{b}{ }^{A A^{\prime}} \delta_{B A^{\prime}} \psi^{B a}+i \epsilon^{a b c} \psi^{A c}\right) .
$$

We use here the two-component spinor notation 13], in which the Infeld-van der Waerden symbols $\sigma_{b}{ }^{A A^{\prime}}$ represent the components of the matrices $\frac{1}{\sqrt{2}} \tau^{b}$.

We now determine the form of the quantum supersymmetry generators for $N=1$ supergravity subject to these conditions. Rewriting the fields in terms of the local coordinate basis as $\psi^{A}=\psi^{A}{ }_{i} \tilde{\boldsymbol{d}} x^{i}$ and $\tilde{\boldsymbol{e}}^{a}=e^{a}{ }_{i} \tilde{\boldsymbol{d}} x^{i}$, and defining $e^{A A^{\prime}}{ }_{i} \equiv \sigma_{a}{ }^{A A^{\prime}} e^{a}{ }_{i}$, these generators have the form [13, 14]

$$
\bar{S}_{A^{\prime}}=\epsilon^{i j k} e_{A A^{\prime} i} D_{j} \psi_{k}^{A}+\frac{\kappa^{2}}{2} \psi_{i}^{A} \frac{\delta}{\delta e^{A A^{\prime}}}
$$


and

$$
S_{A}=D_{i} \frac{\delta}{\delta \psi^{A}{ }_{i}}+\frac{\kappa^{2}}{2} \frac{\delta}{\delta e^{A A^{\prime}}{ }_{i}}\left(D^{B A^{\prime}}{ }^{j i} \frac{\delta}{\delta \psi^{B}{ }_{j}}\right)
$$

where $\kappa^{2}=8 \pi$ and $D_{i}$ denotes the torsion-free spatial covariant derivative. An expression for the kernel $D^{A A^{\prime}}{ }_{j i}$ is given in 13 .

Supersymmetry imposes on the wave function $\Psi\left(e^{A A^{\prime}}{ }_{i}, \psi^{A}{ }_{i}\right)$ the constraints

$$
\bar{S}_{A^{\prime}} \Psi=0=S_{A} \Psi
$$

Together with the angular momentum constraints, these ensure that all the remaining constraints of the theory are also satisfied. For the mini-superspace model discussed here, it will be enough to consider the zero-fermion state, which is annihilated by the operators $\frac{\delta}{\delta \psi^{A}}$ and whose wave function $\Psi_{0}$ therefore depends only on the 3-geometry.

For this state, the $S_{A}$ constraint is satisfied automatically. Following D'Eath [10], we evaluate the other supersymmetry constraint subject to the homogeneity conditions. After some algebra, the $\bar{S}_{A^{\prime}}$ constraint may be rewritten as

$$
-\operatorname{det}\left[\omega^{p}{ }_{i}\right]\left(\frac{1}{2} e_{A A^{\prime}{ }_{s}} \delta^{s r} \psi^{A}{ }_{r}+\frac{i \lambda}{\sqrt{2}} \epsilon^{p q r} e_{B A^{\prime} p} \mathcal{O}_{q}{ }^{a} \sigma_{a}{ }^{B B^{\prime}} \delta_{A B^{\prime}} \psi^{A}{ }_{r}\right) \Psi_{0}=\frac{\kappa^{2}}{2} \psi^{A}{ }_{r} \frac{\delta \Psi_{0}}{\delta e^{A A^{\prime}}{ }_{r}}
$$

where $e^{A A^{\prime}}{ }_{p} \omega^{p}{ }_{i}=e^{A A^{\prime}}{ }_{i}$ and $\psi^{A}{ }_{p} \omega^{p}{ }_{i}=\psi^{A}{ }_{i}$. Here we have introduced a parameter $\lambda$ which is 1 for our ansatz (2), and which vanishes for the ansatz used by D'Eath. We note that both terms in the bracket of eq. (3) have been combined in the single term proportional to $\lambda$.

Cancelling the factors of $\psi_{r}^{A}$ which appear on both sides, and integrating over the whole of $\mathcal{S}^{3}$ we obtain

$$
\frac{\kappa^{2}}{2} \frac{\partial\left(\ln \left|\Psi_{0}\right|\right)}{\partial e_{r}^{A A^{\prime}}}=(4 \pi)^{2}\left(\frac{1}{2} e_{A A^{\prime} s} \delta^{r s}+\frac{i \lambda}{\sqrt{2}} \epsilon^{p q r} e_{B A^{\prime} p} \mathcal{O}_{q}{ }^{a} \sigma_{a}{ }^{B B^{\prime}} \delta_{A B^{\prime}}\right)
$$

If the dreibein has the form given in (1) then this expression can be integrated to give

$$
\Psi_{0}=\text { const } \times \exp \left[-\pi\left(e^{2 \beta_{1}}+e^{2 \beta_{2}}+e^{2 \beta_{3}}-2 \lambda e^{\beta_{1}+\beta_{2}}-2 \lambda e^{\beta_{2}+\beta_{3}}-2 \lambda e^{\beta_{3}+\beta_{1}}\right)\right] .
$$


For $\lambda=0$ this is the solution first given in [15] and [6], and later derived in [10] and [11] by the same technique as used here. The $\lambda=0$ solution is now commonly referred to as the wormhole state, as its exponent defines a Euclidean HamiltonJacobi action which gives rise to Riemannian 4-geometries which are asymptotically flat but become singular as the scale parameter $\exp \left(\beta_{1}+\beta_{2}+\beta_{3}\right)$ approaches zero [16, 17]. The latter property forbids the interpretation of this solution as a HartleHawking state, as noted by D'Eath [10].

On the other hand, our new homogeneity condition corresponds to the choice $\lambda=$ 1. In this case our solution (4) has all the features of a Hartle-Hawking state. (This solution has been previously obtained from an $N=2$ supersymmetric extension of the bosonic Wheeler DeWitt equation for Bianchi IX minisuperspace [8].) In particular, when restricted to the isotropic case $\beta_{1}=\beta_{2}=\beta_{3}=\alpha, \Psi_{0}$ grows with $\alpha$ like $\exp \left(3 \pi e^{2 \alpha}\right)$ which is a well-known feature of the Hartle-Hawking state in isotropic Bianchi IX models [3]. Furthermore, by using the exponent of (4) as a Euclidean Hamilton-Jacobi action, one generates a 1-parameter family of 3-metrics on $\mathcal{S}^{3}$ which remain regular for small scale parameters [16, 18].

We note also that both states $\lambda=0,1$ are normalisable for fixed $\alpha$ when integrated over $\beta_{+}=\frac{1}{6}\left(\beta_{1}+\beta_{2}-2 \beta_{3}\right)$ and $\beta_{-}=\left(\beta_{1}-\beta_{2}\right) / 2 \sqrt{3}$, thus allowing a straightforward probabilistic interpretation.

Let us finally remark on solutions in the other fermion sectors. As was first shown for the Bianchi I model in [9] and later for Bianchi IX models with $\lambda=0$ in [10, 7], the constraint of Lorentz invariance, automatically satisfied by $\Psi_{0}$ which depends only on the 3-geometry, rules out states in any but the empty and filled fermion sectors. The same must happen in the $\lambda=1$ case considered here.

Turning to the wave function $\Psi_{f}$ in the filled sector, we can use the duality between particles and holes to conclude that $\Psi_{f} \sim \Psi_{0}^{-1}$. Because the states $\Psi_{0}$ are 
normalisable for fixed $\alpha$, the states $\Psi_{f}$ are not; they diverge for infinite anisotropies and must therefore be disregarded [6].

In conclusion, we have shown that imposing the supersymmetry constraints for $N=1$ supergravity on Bianchi IX 3-geometries and spinors with the homogeneity property (22) yields a unique quantum state with the properties specified by the no-boundary proposal of Hartle and Hawking.

\section{Acknowledgements}

This work was supported by a Sydney University Research Grant, and by the Deutsche Forschungsgemeinschaft through the Sonderforschungsbereich 237 "Unordnung und Grosse Fluktuationen". R.G. wishes to thank the School of Mathematics at the University of Sydney for hospitality, and to acknowledge useful discussions with Peter D'Eath.

\section{References}

[1] B.S. DeWitt Phys. Rev. D160, 1113 (1967)

[2] C.W. Misner, in "Magic without Magic", edited by J. Klauder (Freeman, San Francisco 1972)

[3] J.B. Hartle \& S.W. Hawking Phys. Rev. D28 2960 (1983)

[4] A. Macías, O. Obregón \& M.P. Ryan Jr Class. Quantum Grav. 41477 (1987)

[5] P.D. D'Eath \& D.I. Hughes Phys. Lett. B 214498 (1988); Nucl. Phys. B 378 $381(1991)$

[6] R. Graham Phys. Rev. Lett. 671381 (1991) ; Phys. Lett. 277B 393 (1992)

[7] R. Graham Phys. Rev. D48, 1602 (1993) 
[8] J. Bene \& R. Graham "Supersymmetric Homogeneous Quantum Cosmologies Coupled to a Scalar Field", preprint 1993

[9] P.D. D'Eath, S.W. Hawking \& O. Obrégon Phys. Lett. 300B 44 (1993)

[10] P.D. D'Eath, Phys. Rev. D48, 713 (1993)

[11] M. Asano, M. Tanimoto \& N. Yoshino, "Supersymmetric Bianchi class A models", preprint 1993

[12] I.M. Benn \& R.W. Tucker, "An Introduction to Spinors and Geometry" (IOP Publishing 1987)

[13] P.D. D'Eath Phys. Rev. D29 2199 (1984)

[14] Here we correct a sign error in the second term on the right-hand side of eq. (4.6) of [13] which was taken over in [9, 11]. In order not to obscure the comparison with refs. [9, 11] we shall compensate for this change of sign by choosing $\operatorname{det}\left[\omega^{p} i\right]<0$.

[15] V. Moncrief and M.P. Ryan Jr. Phys. Rev. D44 2375 (1991)

[16] G.W. Gibbons \& C.N. Pope Comm. Math. Phys. 66267 (1979)

[17] N.A. Belinsky, G.W. Gibbons, D.N. Page \& C.N. Pope Phys. Lett. 76B 433 (1978)

[18] M. Atiyah \& N. Hitchin, "The Geometry and Dynamics of Magnetic Monopoles" (Princeton University Press, 1988) 